\title{
How Artificial Intelligence May Impact Your Job
}

\author{
Gianluca P. M. VIRGILIO ${ }^{\mathrm{a}, 1}$ and Manuel Ernesto PAZ LÓPEZ ${ }^{\mathrm{b}}$ \\ a Universidad Católica Sedes Sapientiae, Nueva Cajamarca, Peru \\ ${ }^{\mathrm{b}}$ Universidad Nacional de Tumbes, Tumbes, Peru
}

\begin{abstract}
Recent developments of Artificial Intelligence, Machine Learning and Big Data technologies are posing a threat to white-collar workers and managers alike. Whereas until a few years ago only manual jobs were at risk of replacement, nowadays there is no guarantee that intellectual jobs will remain in human's hands for long. Hopes that new technologies will generate more new jobs than those lost are vanishing. The new challenge faced by human workers is made up of entities that display more intelligence, whether real or just simulated, than them. We, as humans, need to invent new strategies to survive in a job market that seems ready, in one or two generations, to get rid of us. This paper identifies the main issues around intelligence and provides hints about what intelligence imitation/simulation cannot do that human intelligence can. It also discusses aspects of creativity, allegedly a human-only skill that looks like the most promising area in which human development may progress, reducing risk of silicon replacement.
\end{abstract}

Keywords. Artificial Intelligence, Big Data, Machine Learning, unemployment

\section{Introduction}

The more and more ubiquitous presence of Artificial Intelligence (AI) in business processes is going to impact firms at all levels. The origins of the Fourth Industrial Revolution date back to the 1960s with introduction of mechanical robots in the automotive manufacturing industry to replace blue collar workers in unsafe and unhealthy tasks. Since then, progression towards the unmanned workplace has been abrupt and irregular. However, recent advances in the AI arena seem to have paved the way to the future.

The famous Turing Test defines AI as a machine behavior that an independent evaluator would be unable to distinguish from a human one [1]. Many years have passed since the original formulation of the Turing Test and the problem seems to have turned around [2]. Currently it is up to humans to simulate AI behavior if they want to safeguard their jobs against silicon competitors. Not only can blue collar workers now be replaced, but even physicians, engineers, teachers, and managers are not guaranteed to keep their job against AI carrying out similar tasks with better performance.

Several categories of low-skill workers are now at risk to lose their job to AI. Hopes they will re-train into AI scientists to find another job are thin; once fired, they are likely to face life-long unemployment. It may be argued that this is the price to pay to progress

\footnotetext{
${ }^{1}$ Corresponding Author: Gianluca P. M. VIRGILIO, FCEC, Universidad Católica Sedes Sapientiae, Nueva Cajamarca, Rioja, San Martín, Peru. ORCID: 0000-0002-1616-0557. E-mail: consultgv@gmail.com.
} 
and it is true that their children who studied engineering or business management may fill the newly-created positions (the 'digital divide'). Yet, because of ever-improving AI capabilities, they may follow their parents' destiny in one decade or two. Technology is progressing (at worst) linearly whereas human capabilities are improving (at best) asymptotically. The AI bar keeps moving upward and mankind may not keep pace.

In the last few decades, managers and other highly skilled professionals have been at the forefront of industrial automation, only to find themselves on the brink of being ousted by the Artificial Intelligence Revolution, where the intelligence to be replaced is theirs. It is no longer enough to label a job as 'intellectual' or 'cognitive' to make it immune from the risk of falling prey to AI.

\section{Artificial Activities}

\subsection{AI in manufacturing}

The First Industrial Revolution began with the deployment of mechanical looms in textile factories. Since then, automation in the clothing industry has progressed a long way. Yang et al. [3] argue as today AI-based systems make decisions on production, marketing, choice of fabrics, colors, shapes. The proposed solutions adhere most to customer's preferences, selected on the basis of advanced statistical techniques and Big Data, by retrieving information from a huge number of fashion magazines.

More in general, in the manufacturing industry, AI has proved able to identify the production processes that need improvement. Then, it collects data, executes analyses, identifies causes of poor performance, provides measures for improvement and carries it out [4]. On the supply side, Thomassey and Zeng [5] describe a digital platform consisting of two modules: the first one allows to make decisions about selecting suppliers using multi-criteria techniques while the second one uses a genetic algorithm for optimizing cost and lead time. Finally, Nanda Kumar and Panneerselvam [6] propose an AI-based solution for optimizing the outbound logistic process.

\subsection{AI and MOOCs}

Massive Open Online Courses, Knowledge Management, and Computer-Assisted Learning are terms that have taken on the meaning of flexibility and ease of learning for students [7] and unemployment for teachers, educational programmers and school managers. According to the World Economic Forum report, the day of human teachers being largely replaced by AI is not far away: World Economic Forum [8] includes university and higher education teachers among the most likely redundant jobs in the next future.

\subsection{AI in Medicine}

In medicine, Arslan et al. [9] report of high performance, AI-assisted investigation methods that made early diagnosis of prostate cancer achievable. Additionally, with the aid of AI-driven deep learning, Big Data and statistical techniques it was possible to successfully identify the most appropriate therapeutic intervention for gastroenterological patients [10] or patients with Alzheimer's disease [11]. In spine 
surgery, Buza and Gum [12] present a case of robotic-assisted surgery in which screw size, length and orientation are delegated to robotic technology in order to ensure a minimally invasive approach. In these and many other cases AI has at some extent been replacing physicians' work; more pervasive replacement is expected in the next decade [13].

\subsection{AI and speech content}

A common criticism to Artificial Intelligence applications concerns its lack of contentcomprehension, a core topic in managerial jobs. Gröndahl et al. [14] addressed this issue by detecting hate speech on social networks. They used node-representation learning techniques as anti-fraudster tool in order to identify opinion frauds, groups of fake accounts and search engine poisoning [15]. AI has also been used to combat adversarial perturbations and malicious data manipulation through Big Data and machine learning techniques [16].

\subsection{AI in Human Resources Management}

Klüver and Klüver [17] simulate the behavior of a human brain by implementing input and output neurons, activation functions, learning rules and variable weight values to make decisions about Human Resources (HR) Management and Models of Procedure for planning and performing industrial processes. As long as firms keep employing human workers, human behavior will remain a parameter to be closely monitored within organizations, with motivation being as usual at the forefront of search for performance. Technology-based communication is spreading all over and lack of face-to-face relationships is a serious concern for HR managers. Pratt and Cakula [18] highlight the potential AI has in increasing effectiveness of communications and enhancing motivation, moving one step forward to replacing humans in this area as well.

\subsection{AI in decision-making}

Research is progressing fast in machine-simulation of brain thinking processes by mimicking its internal structure (made of synapses and neurons) via Neural Networks and Deep Learning. This way AI has been used to take charge of decision making tasks by accessing huge databases, making use of sophisticated statistical tools and selecting the solutions most likely to succeed. Intelligence no longer seems to be a human-only capability [19]. An enthusiastic prediction, which actually conceals a gloomy future for managers, states "time will come when the management of the organisation will be entrusted in the hands of the AI system thus automating the entire decision-making process. Decisions will be taken at the speed of light [...]. Managers will be relieved from the day-to-day micro-management" [20, pp.xii-xiii]. Until they will, of course, be relieved from macro-management tasks too. In the meantime, skeptical managers will be shown all details of the AI-based decision-making process through Explainable AI (XAI), where the internal reasoning mechanism of intelligent systems will be kindly presented in a way suitable for even humans to understand [21]. 


\section{Intelligent Activities}

Historically, all industrial revolutions of the past resulted in a net increment of higher paid and more intellectually stimulating jobs. Yet, for the first time in history, mankind faces a competitor. AI displays, or can simulate, more intelligence than us. This leads to a complex question, about the essence of intelligence itself, and whether mere imitation would suffice to replace it. The issue is paramount to the future of human employment since HR managers will hire the entity that demonstrates the best performance, whether flesh-and-blood or silicon. No matter if business goals will be reached via sophisticated human reasoning or brute computing power. As we have seen over the last fifty years, unintelligent tasks can be easily replaced by robots. With support of AI technology managerial tasks can be replaced as well. The 'mythical' Zero-Defect (ZD) target, dream and goal of a bevy of production managers, can now be achieved by their silicon counterparts: Psarommatis and Kiritsis [22] propose a methodology to assess and select the best-fitting approach between product-based and process-based ZD manufacturing. Humans definitely need to mark the difference of their contribution to business results compared to what AI can, or can't, do, if they are to safeguard their jobs.

\subsection{Making use of intelligence}

Although this may sound disappointing, we have to admit that most activities humans execute do not actually involve 'intelligence', according to how we are used to defining it [23]. In most of our daily activities, like walking, washing hands, eating, waiting for the bus, drinking a glass of water, and so on, the actual amount of intelligence we make use of, is small. In all these, and many other cases, our brain reacts to external inputs according to standard protocols: applying intelligence not required. A simple demonstration comes from everyday life. We all, as students or parents, have heard teachers complaining about pupils 'not committing enough'. It means that just applying standard protocols to studying would hardly get a pass. More commitment is required, more dedication, more effort. In a word: more intelligence. Most of the times, average pupils (in their teachers' opinion) do not seem to make enough use of their brain. The same reasoning applies to adults. Most of the times we do not commit enough.

\subsection{Making use of enough intelligence}

Occasionally, for example when studying hard and in several other cases, humans do get involved in less mundane activities than those listed above. In such cases, we use a higher amount of intelligence, yet hardly the amount required to demonstrate our (supposed) superiority to machines. A typical example of an activity that no animal could carry out, and that therefore we consider 'intelligent', is driving a car. Unfortunately, also in this case we are actually behaving rather mechanically, displaying no more intelligence than an AI-driven car does: we receive inputs from our visual system (the human sensors) and apply a set of standard rules. We tend to apply the same approach to decisions at work. With the exception of facing an unfamiliar problem, like studying a new topic, or a brand new situation, we have been trained to apply solutions learned from books or from previous experience. Yet, this is also the approach AI would apply.

By suitably increasing knowledge bases, logical relations between information, and learning from previous attempts in a trial-and-error process, AI and Big Data can mimic (and improve) the human learning mechanism. This is the essence of Machine Learning: 
doing exactly what human children do very easily in early years of their existence with lots of time and application by their instructors. Machines can accomplish this much quicker, at lower cost and to better results.

\section{Countermeasures}

Manager and executives are now facing the problem of retaining their job against silicon competitors that are less prone to errors, act faster, cost less and are more suitable to expand their capabilities. The only reasonable solution is looking for activities that computers, algorithms and AI will never be able to replicate - and extract jobs out of them. In the last few decades, though technology has progressed dramatically, improvements in performing innovative tasks did not match advancements in repetitive or mechanical routines.

\subsection{Non-replaceable activities}

Generation of new ideas, philosophy, negotiation, debates in court, and commercial activity are tasks we do not use computers for. These are activities that need to be invented every time anew. Novel problems require novel solutions; ideas can spring from general principles but must be specific for the problem on the table. Similarly, interacting with humans on the other side, like a judge, a reader, a political or commercial counterpart, cannot be copied-and-pasted from previous experience as is: it requires innovation. Machines can simulate intelligence but they still lack what is known as 'emotional intelligence', the ability to empathize with others. So far AI has not proved able to identify the subtlety of an argument to convince a jury of its own view or a customer about her need to purchase a good or service. Additionally, fact interpretation heavily depends on human sensitivity, and as such it seems unsuitable to machines. Not every aspect of our life seems suitable for automations.

\subsection{Human uniqueness}

The trend seems now clear: whatever can be automated will be. This assigns significant importance to what makes us as humans unique: application of curiosity, humor, empathy, creativity, wisdom, figures of speech, and metaphors [24] among many other human features. Algorithms are not able to simulate or adopt any of the above. Interpretation of the content of a book is another activity that seems for-human-only since it needs a complex mix of knowledge and capability to understand the inexplicit [25]. The nuances required by a human being to understand how to correctly interpret events are by far too ethereal for fitting a, no matter how large, database table.

Despite their huge improvements, computers did not prove able so far to produce something that was not, one way or another, already written in their hardware, software, or stored knowledge. Algorithms never displayed the capability of intuition quantum leap. A stimulating point is algorithm's capability to write another algorithm whose logic is not already included in the former. Creating a solution model from scratch is a basic skill that even beginner programmers can display, but hard to achieve by AI. The reason being that in order to write anything, software or poetry, a certain degree of creativity, or innovation, or intuition is required, and thus far it is not completely clear even to psychologists what such concepts really mean [23]. 
Interpretation of meanings is much more complex and beyond the grasping of any chip, algorithm or database. AI cannot interpret the meaning of books and even short documents. The subtle details of legal language may be hard to grasp by outsiders, let alone Artificial Intelligence. Nevertheless, legal and formal language is used for writing important documents: international agreements and commercial contracts.

\subsection{AI conferences vs. Employment conferences}

Inventing ways in which humans can retain their jobs in an AI-led world should be the main focus of white collar workers, managers and executives. That should be the real hot topic of academic and business conferences. At the contrary, the more news about scientific conferences we hear and more academic papers we read, and the more we realize that they deal with new successes achieved and old hurdles left behind.

A (non-exhaustive) internet search about AI-related conferences in 2019-2021 (up to August) and those programmed in September-December 2021 and in 2022 reveals 81 congresses subdivided as follows: 23 on generic AI, 12 on Computer Vision, 11 on Computer Speech, 9 on Machine Learning, 6 on Big Data, 5 each on Knowledge Management, Robotics and Multimedia, 3 on (AI-related) Health Care and 2 on Neural Networks. On the other side, just 7 conferences (less than 8\%) over the same period were found when using the 'Employment' keyword, 2 of which dealt with employment law (which sounds suspiciously akin to researching new ways for firing human workers in order to make room for intelligent computers!).

To the best of my knowledge this trivial comparison has never been carried out before and although both searches can certainly be improved and extended, it is unlikely that the ratio between the two types of congress will change significantly. A complete change of paradigm is long overdue. We only have our brain to compete in this contest; let's make use of it!

\section{Conclusion}

Impressive technological advancements achieved thanks to AI means humans will have to adapt, once more in Mankind's history, to the newly generated environment, not differently to what happened during the First Industrial Revolution in the XVIII century. Because of the different characteristics of the current industrial revolution, the skills required to remain attractive in the newly generated work environment will be very different from the ones needed so far. It will be the goal of the human race to learn them in a short timeframe. A newly designed course of study will hardly provide the required answer; a much wider effort is called for by academia, business practitioners, politicians, trade unions, and the whole community of white collar workers, including managers and executives. Several unresolved issues are piling up: without a thorough comprehension of what intelligence is, what its unique features are and what can we do to exploit this gift to the maximum, the race for sustainable human employment in the future cannot be won. We need to provide answers to what computers can and will be able to do in the future in order to find alternative applications for humans to carry out - and to earn their living from. The risk in twenty years' time is having eight billion people struggling to get the relatively few jobs available as lawyers, psychologists or salespeople and other jobs requiring soft skills: a very serious discussion on this vital topic is now called for. 
White collar workers, managers and executives will have to be at the forefront of this debate: their future is at stake.

\section{References}

[1] Turing A. Computing machinery and intelligence. Mind, 49:433-460

[2] Hall O. Passing the Turing Test. Graziadio Business Review 21(2)

[3] Yang T, Feng J, Chen J, Dong C, Shi Y, Tao R. A Clothing Recommendation System Based on Expert Knowledge. In: Wong W. (ed.) Artificial Intelligence on Fashion and Textiles. AIFT 2018, Hong Kong

[4] Yang B, He Y, Yin H. Data Analysis and Production Process Control. In: Dolgui A, Bernard A, Lemoine D, von Cieminski G, Romero D. (eds) Advances in Production Management Systems. APMS 2021. Nantes, France. Springer. Cham, Switzerland

[5] Thomassey S, Zeng X. FBD_Bmodel Digital Platform: A Web-Based Application for Demand Driven Fashion Supply Chain. In: Dolgui A, Bernard A, Lemoine D, von Cieminski G, Romero D. (eds) Advances in Production Management Systems. APMS 2021 Nantes, France Springer. Cham, Switzerland

[6] Nanda Kumar S, Panneerselvam R. A Genetic Algorithm-based Artificial Intelligence Solution for Optimizing E-Commerce Logistics Vehicle Routing. In: Suguna SK, Dhivya M, Paiva S. Artificial Intelligence (AI) Recent Trends and Applications. CRC Press. Boca Raton, FL

[7] Botticelli M, Gasparetti F, Sciarrone F, Temperini M. Deep Learning to Monitor Massive Open Online Courses Dynamics. In: De la Prieta F, Gennari R, Temperini M, Di Mascio T, Vittorini P, Kubincova Z, Popescu E, Rua Carneiro D, Lancia L, Addone A (eds). Methodologies and Intelligent Systems for Technology Enhanced Learning, $11^{\text {th }}$ Conference. Springer. Cham, Switzerland

[8] World Economic Forum. The Future of Jobs Report 2020. www.weforum.org

[9] Arslan M, Arslan D, Haznedar B. Training ANFIS Systems With Genetic Algorithm For Diagnosis Of Prostate Cancer. Technological Applied Sciences. 13(4):301-309.

[10] Ruffle JK, Farmer AD, Aziz Q. Artificial Intelligence-Assisted Gastroenterology—Promises and Pitfalls. The American Journal of Gastroenterology. October 2018.

[11] Tang H, Yao E, Tan G, Guo X. A Fast and Accurate 3D Fine-Tuning Convolutional Neural Network for Alzheimer's Disease Diagnosis. In: Zhou Z, Yang Q, Gao Y, Zheng Y (eds.). Artificial Intelligence. ICAI 2018; Jinan, China

[12] Buza J, Gum J. Cortical Screw Fixation. In: Wang MY, Steele III WJ, Urakov T. Advanced Robotic Spine Surgery. CRC Press. Boca Raton, FL

[13] Davenport T, Kirby J. Only Humans Need Apply. Harper Collins, New York

[14] Gröndahl T, Pajola L, Juuti M, Conti M, Asokan N. All You Need is "Love": Evading Hate Speech Detection. AISec'18. Toronto, Canada.

[15] Xu C, Feng Z,Chen Y, Wang M, Wei T. FeatNet: Large-scale Fraud Device Detection by Network Representation Learning with Rich Features. AISec'18. Toronto, Canada

[16] Rieck K. Family Reunion: Adversarial Machine Learning meets Digital Watermarking. In: Multimedia Privacy and Security 2018 (invited keynote). Toronto, Canada. https://doi.org/10.1145/3267357.3267366

[17] Klüver C., Klüver J. Decision Support in Everyday Business Using Self-enforcing Networks. In: Dingli A, Haddod F, Klüver C. (eds.) Artificial Intelligence in Industry 4.0. Springer. Cham, Switzerland

[18] Pratt M, Cakula S. Motivation in a Business Company Using Technology-Based Communication. In Artificial Intelligence in Industry 4.0. Springer. Cham, Switzerland

[19] Finlay R, Takeda H. Reflections on Decision-Making and Artificial Intelligence. In: Braunschweig B, Ghallab M. Reflections on Artificial Intelligence for Humanity. Springer. Cham, Switzerland

[20] Dingli A, Haddod F, Klüver C. Artificial Intelligence in Industry 4.0. Springer. Cham, Switzerland

[21] Mallia N, Dingli A, Haddod F. MIRAI: A Modifiable, Interpretable, and Rational AI Decision Support System. In: Dingli A, Haddod F, Klüver C. (eds.) Artificial Intelligence in Industry 4.0. Springer. Cham, Switzerland

[22] Psarommatis F, Kiritsis D. Comparison Between Product and Process Oriented Zero-Defect Manufacturing (ZDM) Approaches. In: Dolgui A, Bernard A, Lemoine D, von Cieminski G, Romero D. (eds) Advances in Production Management Systems - Artificial Intelligence for Sustainable and Resilient Production Systems. APMS 2021. Nantes, France. Springer. Cham, Switzerland

[23] Purves D. Why Brains Don't Compute. Springer. Cham, Switzerland

[24] Egan Y, Chesley J. Leadership in a Smart Machine Age Lessons on Becoming More Human from the Mind-Brain Revolution. Graziadio Business Review 21(2)

[25] Smith G. The AI Delusion. Oxford University Press. New York 